\title{
Incidência de protocolos de morte encefálica, captações e fatores que influenciam o processo de doação de órgãos em um Complexo Hospitalar Regional
}

\author{
Incidence of brain death protocols, uptakes and factors that influence the organ donation \\ process in a Regional Hospital Complex
}

Incidencia de protocolos de muerte cerebral, captación y factores que influyen en el proceso de donación de órganos en un Complejo Hospitalario Regional

Erick Marques Pinheiro ${ }^{1 *}$, Állef Diego Bonfim de Andrade ${ }^{1}$, Ledne Luiz Dalla Rosa ${ }^{3}$, Bianca Oyola Bicalho $^{3}$, Valério Bortolini ${ }^{3}$, Egberto Luiz Felicio Junior ${ }^{3}$.

\section{RESUMO}

Objetivo: Apontar fatores que segundo os profissionais de saúde envolvidos no cuidar do potencial doador, podem influenciar no processo de captações de órgãos. Além de apresentar dados de incidência de Protocolos de Morte Encefálica, de captações de órgãos ocorridos nos anos de 2017 e 2018 do Complexo Hospitalar Regional de Cacoal (COHREC). Métodos: Pesquisa transversal, de caráter descritivo, com abordagem quantitativa e qualitativa. O levantamento de dados ocorreu por meio de um questionário aplicado aos profissionais atuantes há mais de seis meses na sala vermelha e nas Unidades de Terapia Intensiva (UTI) do COHREC. Resultados: Foi possível constatar que os principais fatores que os profissionais citaram foram a não observação ou não participação de alguma captação de órgão, a falta de profissionais, a falta de capacitação dos profissionais, a falta de equipamentos e a falta de estrutura. Conclusão: Deve-se buscar a qualificação multiprofissional, não só para diagnóstico e manutenção do potencial doador, mas para desenvolvimento de habilidades relacionadas à comunicação entre os profissionais, a comunicação para com os familiares e proporcionar ao profissional apoio psicológico e condições dignas de trabalho.

Palavras-chave: Morte Encefálica, Doação de órgãos e tecidos, Atenção integral a saúde, Educação Continuada.

\begin{abstract}
Objective: Point out factors that according to health professionals involved in the treatment of potential donors, may influence the process of organ procurement. In addition to presenting incidence data of Brain Death Protocols, organ uptake occurred in the years 2017 and 2018 of the Cacoal Regional Hospital Complex (COHREC). Methods: Cross-sectional, descriptive research with quantitative and qualitative approach. The data collection took place through a questionnaire applied to professionals working for more than six months in COHREC's Red Room and Intensive Care Units (ICU). Results: It was found that the main factors mentioned by the professionals were the non-observation or non-participation of any organ uptake, the lack of professionals, the lack of training of professionals, the lack of equipment and the lack of structure. Final Considerations: Multiprofessional qualification should be sought, not only for diagnosis and maintenance of the potential donor, but for the development of skills related to communication between professional, communication with family members and providing the professional with psychological support and decent working conditions.
\end{abstract}

Key words: Brain Death, Organ and tissue donation, Comprehensive health care, Continuing Education.

${ }^{1}$ Complexo Hospitalar Regional de Cacoal (COHREC/HRC/HEURO), Cacoal - RO.

*E-mail: erickmp17@hotmail.com

2Secretaria de Estado da Saúde (SESAU), Cacoal - RO.

SUBMETIDO EM: 11/2019

ACEITO EM: 12/2019

PUBLICADO EM: 2/2020 


\section{RESUMEN}

Objetivo: Señale factores que, según los profesionales de la salud involucrados en el tratamiento de donantes potenciales, pueden influir en el proceso de obtención de órganos. Además de presentar los datos de incidencia de los Protocolos de Muerte Cerebral, la absorción de órganos se produjo en los años 2017 y 2018 del Complejo Hospitalario Regional Cacoal (COHREC). Métodos: Investigación descriptiva transversal con enfoque cuantitativo y cualitativo. La recopilación de datos se realizó a través de un cuestionario aplicado a profesionales que trabajan durante más de seis meses en la Sala Roja y las Unidades de Cuidados Intensivos (UCl) de COHREC. Resultados: Se encontró que los principales factores mencionados por los profesionales fueronla no obsevación o la no participación de la captación de órganos, la falta de profesionales, la falta de capacitación de los profesionales, la falta de equipos y la falta de estructura. Consideraciones Finales: Se debe buscar la calificación multiprofesional, no solo para el diagnóstico y el mantenimiento del donante potencial, sino para el desarrolhode habilidades relacionadas con la comunicación entre profesionales, la comunicación con los miembros de la familia y la provisión de apoyo psicológico y condiciones de trabajo dignas para el profesional.

Palabras clave: Muerte Cerebral, Donación de órganos y tejidos, Atención integral de salud, Educación Contínua.

\section{INTRODUÇÃO}

Morte encefálica (ME) é definida como a perda completa e irreversível das funções do córtex e do tronco cerebral, de causa conhecida e constatada de modo indiscutível, caracterizada por coma aperceptivo, com ausência de resposta motora supraespinhal e apnéia (HIRSCHHEIMER MR, 2016).

A ME representa o estado clínico irreversível em que as funções cerebrais (telencéfalo e diencéfalo) e do tronco encefálico estão irremediavelmente comprometidas (MORATO EG, 2009). Os procedimentos para determinação da ME deverão ser realizados em todos os pacientes em coma não perceptivo e apnéia, independentemente da condição de doador ou não de órgãos (CFM, 2017).

As causas mais comuns de morte encefálica (ME) são: traumatismo cranioencefálico e acidente vascular encefálico. Seu diagnóstico é essencialmente clínico e os familiares que estiverem em companhia do paciente ou que tenham oferecido meios de contato serão obrigatoriamente informados do início do procedimento de reconhecimento. Diagnosticar a morte com o máximo de antecedência possibilita preservar tecidos e órgãos (CIOATTO RM e PINHEIRO AAG, 2017).

A identificação e manutenção precoce do possível doador refletem de forma significativa na qualidade dos órgãos que poderão ser doados. Pacientes com diagnóstico neurológico grave irreversível, apresentando pupilas fixas e bilaterais, com Escala de Coma Glasgow igual a 3, deverão ser considerados em alto grau de suspeita de diagnóstico de morte encefálica (WESTPHAL GA, et al., 2016).

São considerados critérios para a abertura do protocolo de morte encefálica: o coma arreativo e aperceptivo, com Glasgow 3; o paciente deve estar em ventilação mecânica; a causa do coma deve ser conhecida, por meio da história clínica, exame físico e exames complementares; as estruturas vitais do encéfalo devem estar com lesão irreversível diagnosticada por método de imagem; deve-se excluir o uso de drogas depressoras do Sistema Nervoso Central, com níveis capazes de causar o coma e mimetizar a morte encefálica; ausência de hipotermia; não apresentar severos distúrbios metabólicos ácido-básico e/ou eletrolíticos capazes de levar ao coma e mimetizar a morte encefálica e não estar com hipotensão arterial (CUNHA DSP, et al., 2018).

Para a confirmação do diagnóstico de ME é imprescindível realizar dois exames neurológicos, que devem ser efetuados por dois médicos diferentes, sendo um deles obrigatoriamente neurologista ou neurocirurgião. É obrigatória também a realização de exame complementar para comprovar a ausência de atividade elétrica e metabólica encefálica ou de perfusão sanguínea encefálica. Em seguida, notifica-se o potencial doador à Organização de Procura de Órgãos (OPO), que irá avaliar as condições clínicas e realizar a entrevista familiar quanto à doação (JACAÚNA RO, et al., 2017). 
Tanto a ME quanto as lesões cerebrais afetam o equilíbrio do organismo, no entanto, a alteração desses sistemas pode ser revertida por meio de um manejo minucioso e rigoroso do paciente (FAGIOLI FGD e BOTONI FA, 2009).

Nos últimos anos, o avanço das técnicas de preservação de órgãos permitiu aumentar o número de doadores e garantir o funcionamento adequado dos órgãos até a captação e o transplante. Nesse sentido, muitos países reconhecem que o princípio da doação de órgãos deve ser um dos componentes dos cuidados de final de vida de todos os doentes que morrem em uma unidade de cuidados intensivos (DA EIRA CSL e TRINDADE MI, 2018).

Durante o processo de doação é fundamental a avaliação rigorosa do potencial doador, bem como, o cuidado, ao qual se prioriza ser em uma UTI, visto que é um setor que possui condições de proporcionar ao paciente atenção com equipe especializada constantemente, monitorização, materiais especifícos, além das tecnologias que auxiliam no tratamento. Outro ponto a se destacar, é a atuação dos profissionais de outros setores, como por exemplo, as Comissões Intra-Hospitalares de Doação de Órgãos e Tecidos para Transplantes (CIHDOTT) e a Central de Transplantes que contribuem com o andamento do protocolo, as avaliações e o cuidado ao potencial doador (MAGALHÃES ALP, et al., 2017).

A doação de órgãos no país é regulamentada pela Legislação Brasileira sobre doação de órgãos humanos, pela Lei o 9.434, de 4 de fevereiro de 1997. Contudo, e apesar da legislação pertinente, o que se identifica no país é uma elevada demanda de pessoas a espera de um doador de órgãos compatível e, por outro lado, uma baixa oferta de possíveis doadores potenciais (REZENDE LBO, et al., 2015).

O Brasil possui hoje $90 \%$ dos transplantes realizados pelo Sistema Único de Saúde (SUS), ocorrendo cerca de 19.000 transplantes de órgãos por ano. Apesar do crescente número de transplantes, a necessidade de doação também vem aumentando, porém em escala maior que a efetivação dela, levando à escassez de órgãos e favorecendo a ampliação da lista de espera (RIBEIRO SCP, et al., 2017).

A doação e alocação de órgãos é um processo trabalhoso e delicado que depende da confiança da população no sistema e do comprometimento dos profissionais de saúde no diagnóstico de morte encefálica. O Brasil é o segundo país do mundo em número de transplantes e, para consolidar essa conquista, é crucial a atuação do Ministério da Saúde, dos governos estaduais, das entidades e profissionais de saúde em todo o processo de doação e transplantes (ABTO, 2017).

No que se trata do quantitativo de doadores efetivos, no ano de 2017 o Brasil apresentou um aumento de $14 \%$ em relação a 2016 , correspondendo a 3.415 doadores e uma taxa de 16,6 por milhão da população (PMP). Tal número se torna baixo quando comparado a outros países, como é o caso da Espanha que no mesmo ano atingiu uma taxa de 46,9 PMP. Diante deste contexto, ressalta-se que o país ainda apresenta significativa mudança desse indicador entre as regiões. Por exemplo, a região Sul e Norte que possuem uma taxa de 34,1 e 3,9 PMP de doadores efetivos, respectivamente (ABTO, 2017; COELHO GHF e BONELLA AE, 2019).

No relatório de Registro Brasileiro de Transplantes verificou-se que a região Sul no ano de 2018 apresentou uma taxa de 35,9 PMP de doadores, sendo dez vezes acima da região Norte (3,6 PMP). Destaca-se que no ano de 2008 a 2012 a região Norte havia passado de 0,6 doadores (PMP) para 3,7 PMP. No entanto, observa-se que nos últimos anos esse número não cresceu, estagnando numa taxa menor que a média do país, para ser mais específico, essa taxa encontra-se cinco vezes menor que a nacional (ABTO, 2018).

No ano de 2017, o Brasil realizou 8.642 transplantes, dentre eles: coração, fígado, pâncreas, pulmão, rins e córnea. Nesse mesmo ano foram adicionadas 10.565 pessoas a lista de espera, evidenciando mais uma vez o desequilíbrio entre a oferta e a demanda (ABTO, 2017; COELHO GHF e BONELLA AE, 2019).

Desse modo, essa pesquisa se faz importante por demonstrar a realidade de um hospital no interior da região Norte sobre o processo de doação de órgãos, visto que os mecanismos e as normas para captar órgãos ainda se encontram com um lento progresso. Assim, essa pesquisa tem por objetivo apontar fatores 
que segundo os profissionais de saúde envolvidos no cuidar do potencial doador, podem influenciar no processo de captações de órgãos. Além de apresentar dados de incidência de Protocolos de Morte Encefálica, de captações de órgãos e identificar os órgãos captados nos anos de 2017 e 2018 do Complexo Hospitalar Regional de Cacoal (COHREC).

\section{MÉTODOS}

Trata-se de uma pesquisa transversal, de caráter descritivo, abordagem quantitativa e qualitativa. A pesquisa de campo ocorreu no COHREC que é composto pelo Hospital de Urgência e Emergência de Cacoal (HEURO) e Hospital Regional de Cacoal (HRC), ambos hospitais de referência no interior do Estado, constituindo a Macro região II de Rondônia. Dentro do complexo, elencou-se os seguintes setores: Sala Vermelha e Extensão da Sala Vermelha do HEURO e nas Unidades de Terapia Intensiva (UTI) 1 e 2 do HRC. As demais informações foram coletadas junto ao Serviço de Arquivo Médico e Estatística (SAME) e a Comissão Intra-Hospitalar de Doação de Órgãos e Tecidos para Transplante (CIHDOTT).

A pesquisa de campo levantou dados, por meio de um questionário dividido em duas partes, a primeira se tratava dos dados sociodemográficos dos participantes, e a segunda possuía 10 perguntas de múltipla escolha, elaboradas pelo próprio pesquisador, abordando fatores relacionados ao contexto de captações de órgãos, como: a participação em Protocolos de Morte Encefálica, a capacitação sobre Protocolo de Morte Encefálica e captações de órgãos, a falta de equipamentos, entre outros.

Participaram da pesquisa todos os profissionais de nível superior ou técnico com mais de seis meses de atuação nos setores citados acima, que aceitaram colaborar com a pesquisa por meio da assinatura do Termo de Consentimento Livre e Esclarecido (TCLE). Foram excluídos da pesquisa os profissionais que estavam de férias, licença prêmio, afastamento e atestado médico durante o período de coleta de dados. Além daqueles que não aceitaram assinar o TCLE.

Após a coleta de dados entrou-se em contato com os responsáveis do Serviço de Arquivo Médico e Estatística (SAME) e da CIHDOTT para levantamento dos dados relacionados ao quantitativo de protocolos de morte encefálica, desfechos, captações e quais os órgãos captados no COHREC nos últimos dois anos. Os dados foram consolidados em base constituída nos programas Microsoft Office Word® e Microsoft Office Excel® versão 2010, posteriormente analisados e discutidos conforme a literatura pertinente.

A presente pesquisa seguiu as recomendações das Normas de Pesquisa Envolvendo Seres Humanos do Conselho Nacional de Saúde (Res. CNS 466/12), sendo realizada após aprovação pelo Comitê de Ética em Pesquisa (CEP), por meio da CAAE 97094918.7.0000.5298 e do parecer 2.879.889 (BRASIL, 2012).

\section{RESULTADOS}

Responderam o questionário 85 (oitenta e cinco) indivíduos, 33 (38,8\%) do sexo masculino e 52 (61,2\%) do sexo feminino, com as faixas etárias dos 20 a 29 anos com o total de $14(16,5 \%)$ indivíduos, dos 30 a 39 anos com o total de $40(47,1 \%)$ indivíduos, dos 40 a 49 anos com o total de $26(30,6 \%)$ indivíduos e 50 ou mais anos com o total de $5(5,9 \%)$ indivíduos, todos profissionais de saúde (médicos, enfermeiros, fisioterapeutas, técnicos de enfermagem e outros) do COHREC com mais de 6 meses de prestação de serviços, que estavam lotados na Sala Vermelha e Extensão da Sala Vermelha do HEURO e UTI's 1 e 2 do HRC. Onde $43(50,6 \%)$ profissionais eram da Sala Vermelha e Extensão da Sala Vermelha do HEURO e 42 (49,4\%) profissionais das UTI's 1 e 2 do HRC (Tabela 1).

Segundo o questionário respondido pelos profissionais, na pergunta 1, 60 (70,6\%) profissionais já observaram ou participaram de algum Protocolo de ME, 24 (28,2\%) responderam que não observaram ou não participaram. Na pergunta 2, $14(16,5 \%)$ profissionais já observaram ou participaram de alguma captação de órgão e 71 (83,5\%) não observaram ou não participaram. Na pergunta 3, 69 (81,2\%) profissionais acreditam que faltam profissionais para o cuidado do potencial doador, $10(11,8 \%)$ acreditam que não faltam e $6(7,1 \%)$ não souberam opinar. 
Tabela 1 - Distribuição de frequências relativa e absoluta quanto aos dados sociodemográficos dos participantes da pesquisa.

\begin{tabular}{|c|c|c|}
\hline Variáveis & $N=85$ & $\%$ \\
\hline \multicolumn{3}{|l|}{ Sexo } \\
\hline Masculino & 33 & 38,8 \\
\hline Feminino & 52 & 61,2 \\
\hline \multicolumn{3}{|l|}{ Faixa etária } \\
\hline 20 a 29 & 14 & 16,5 \\
\hline 30 a 39 & 40 & 47,1 \\
\hline 40 a 49 & 26 & 30,6 \\
\hline 50 ou mais & 5 & 5,9 \\
\hline \multicolumn{3}{|l|}{ Tempo de serviço } \\
\hline$<1$ ano & 9 & 10,6 \\
\hline 1 a 5 anos & 53 & 62,4 \\
\hline 5 a 10 anos & 21 & 24,7 \\
\hline$>10$ anos & 2 & 2,4 \\
\hline \multicolumn{3}{|l|}{ Profissão } \\
\hline Médico & 12 & 14,1 \\
\hline Enfermeiro & 14 & 16,5 \\
\hline Técnico de Enfermagem & 45 & 52,9 \\
\hline Outros* & 14 & 16,5 \\
\hline
\end{tabular}

*Assistente social, fisioterapeuta, fonoaudiólogo, psicólogo e terapeuta ocupacional

Fonte: Pinheiro EM, et al., 2018.

Na pergunta 4, $18(21,2 \%)$ profissionais acreditam que os profissionais responsáveis pelo cuidar do potencial doador estão capacitados para esta responsabilidade, $59(69,4 \%)$ acreditam que os profissionais não estão capacitados, destes $8(9,4 \%)$ não souberam opinar.

Na pergunta 5, $71(83,5 \%)$ dos profissionais não receberam nenhuma capacitação sobre Protocolo de ME e captações de órgãos. Grande parte dos entrevistados 56 (65,9\%) acreditam que faltam equipamentos para o melhor cuidado do potencial doador, bem como a falta de estrutura (espaço físico) $52(61,2 \%)$.

Com relação a pergunta $8,78(91,8 \%)$ profissionais acreditam que uma instabilidade hemodinâmica do potencial doador pode dificultar uma captação de órgão, $1(1,2 \%)$ acredita que não e $6(7,1 \%)$ não souberam opinar. Na pergunta $9,79(92,9 \%)$ profissionais gostariam de fazer uma capacitação sobre captações de órgãos. Na pergunta 10, todos os entrevistados concordam que tendo mais profissionais capacitados, o cuidar do potencial doador será diferenciado (100\%) (Tabela 2). 
Tabela 2 - Distribuição das freqüências absolutas e relativas segundo os profissionais de saúde do COHREC, abordando fatores relacionados ao contexto de Protocolos de ME e captações de órgãos.

\begin{tabular}{|c|c|c|c|c|c|c|}
\hline \multirow{2}{*}{ Perguntas } & \multicolumn{2}{|c|}{ Sim } & \multicolumn{2}{|c|}{ Não } & \multicolumn{2}{|c|}{ Não sei opinar } \\
\hline & $\mathbf{N}$ & $\%$ & $\mathbf{N}$ & $\%$ & $\mathbf{N}$ & $\%$ \\
\hline $\begin{array}{l}\text { 1) Você já observou ou participou de algum } \\
\text { Protocolo de Morte Encefálica? }\end{array}$ & 60 & 70,6 & 24 & 28,2 & 1 & 1,2 \\
\hline $\begin{array}{l}\text { 2) Você já observou ou participou de alguma } \\
\text { captação de órgão? }\end{array}$ & 14 & 16,5 & 71 & 83,5 & 0 & 0,0 \\
\hline $\begin{array}{l}\text { 3) Você acredita que faltam profissionais para } 0 \\
\text { cuidado do potencial doador? }\end{array}$ & 69 & 81,2 & 10 & 11,8 & 6 & 7,1 \\
\hline $\begin{array}{l}\text { 4) Você acredita que os profissionais responsáveis } \\
\text { pelo cuidar do potencial doador estão capacitados } \\
\text { para esta responsabilidade? }\end{array}$ & 18 & 21,2 & 59 & 69,4 & 8 & 9,4 \\
\hline $\begin{array}{l}\text { 5) Você já recebeu alguma capacitação sobre } \\
\text { Protocolo de Morte Encefálica e captações de } \\
\text { órgãos? }\end{array}$ & 13 & 15,3 & 71 & 83,5 & 1 & 1,2 \\
\hline $\begin{array}{l}\text { 6) Você acredita que faltam equipamentos para o } \\
\text { melhor cuidado do potencial doador? }\end{array}$ & 56 & 65,9 & 18 & 21,2 & 11 & 12,9 \\
\hline $\begin{array}{l}\text { 7) Você acredita que falta estrutura (espaço físico) } \\
\text { para o melhor cuidado do potencial doador? }\end{array}$ & 52 & 61,2 & 25 & 29,4 & 8 & 9,4 \\
\hline $\begin{array}{l}\text { 8) Você acredita que uma instabilidade } \\
\text { hemodinâmica do potencial doador pode dificultar } \\
\text { uma captação de órgão? }\end{array}$ & 78 & 91,8 & 1 & 1,2 & 6 & 7,1 \\
\hline $\begin{array}{l}\text { 9) Você gostaria de fazer uma capacitação sobre } \\
\text { captações de órgãos? }\end{array}$ & 79 & 92,9 & 4 & 4,7 & 2 & 2,4 \\
\hline $\begin{array}{l}\text { 10) Você acredita que tendo mais profissionais } \\
\text { capacitados, o cuidar do potencial doador será um } \\
\text { diferencial? }\end{array}$ & 85 & 100,0 & 0 & 0,0 & 0 & 0,0 \\
\hline
\end{tabular}

Fonte: Pinheiro EM, et al., 2018.

No que se refere ao contexto dos Protocolos de ME, no COHREC foram abertos 27 protocolos em 2017 e 21 em 2018, totalizando 48 protocolos. Destes, 33 foram concluídos, correspondendo a 68,75\% dos casos. Observa-se que nos últimos dois anos manteve-se próximo o quantitativo de protocolos fechados (Tabela 3). 
Tabela 3 - Número de protocolos realizados no COHREC nos anos de 2017 e 2018.

\begin{tabular}{lccccccc}
\hline \multirow{2}{*}{ Protocolos } & \multicolumn{7}{c}{ COHREC } \\
\cline { 2 - 8 } & \multicolumn{2}{c}{$\mathbf{2 0 1 7}$} & $\mathbf{2 0 1 8}$ & \multicolumn{2}{c}{ TOTAL } \\
\cline { 2 - 7 } & $\mathbf{N}$ & $\%$ & $\mathbf{N}$ & $\%$ & $\mathbf{N}$ & $\%$ \\
\hline Fechados & 17 & 63,0 & 16 & 76,2 & 33 & 68,75 \\
Não-fechados $^{*}$ & 10 & 37,0 & 5 & 23,8 & 15 & 31,25 \\
\hline Total & 27 & 100,0 & 21 & 100,0 & 48 & 100,0 \\
\hline
\end{tabular}

*Parada cardiorrespiratória antes do fechamento do protocolo.

Fonte: Pinheiro EM, et al., 2018. Baseado em RONDÔNIA, 2018.

Quanto as captações no COHREC, no ano de 2017 ocorreram 7 captações e em 2018 ocorreram 25. Quanto aos órgãos captados no COHREC, no ano de 2017 foram captados rins e córneas, e no ano de 2018 foram captados rins, córneas e fígado. Rondônia possui equipe apenas para transplantes de rins e córneas, mas a captação ocorrida em 2018 do fígado foi proporcionada por uma equipe de transplantes proveniente do Estado do Acre.

\section{DISCUSSÃO}

Quanto ao perfil sociodemográfico dos participantes da pesquisa predominou a equipe de enfermagem correspondendo a $69,4 \%$, sexo feminino $(61,2 \%)$, com faixa etária de 30 a 39 anos $(47,1 \%)$ e que atuam há pelo menos cinco anos (62,4\%). Tais características corroboram com uma pesquisa realizada a nível nacional na qual investigou o perfil desses profissionais de enfermagem chegando à conclusão que no Brasil a equipe de enfermagem é predominantemente feminina $(85,1 \%)$, faixa etária menor que 40 anos (61,7) (COFEN, 2013).

Apesar de $70,6 \%$ dos participantes já terem observado ou participado do protocolo de ME, a maioria $(83,5 \%)$ afirma não ter recebido uma capacitação sobre o protocolo de ME e captações de órgãos. Tais resultados reforçam o que Freire ILS, et al. (2014) concluiu em seu estudo, dizendo que o conhecimento dos profissionais de saúde sobre o diagnóstico de ME e manutenção ao potencial doador é falho, se fazendo necessário educação permanente sobre o tema com o intuito de crescer a oferta de órgãos/tecidos para transplantes.

Estudos apontam que apenas um em cada oito potenciais doadores é, oficialmente, notificado ao sistema de captação de órgãos. Entre as principais barreiras à notificação estão: desconhecimento e/ou desinteresse por parte da equipe e a sobrecarga de trabalho dos profissionais (CASTELLI I, et al., 2018).

Quando questionados sobre o desejo de fazer uma capacitação sobre captações de órgãos, 92,9\% responderam sim, e a unanimidade (100\%) acredita que a capacitação profissional será um diferencial para o cuidado com o potencial doador. O conhecimento de todo o processo, assim como, a sua execução efetiva proporciona a captação de órgãos e tecidos com mais segurança e qualidade, potencializando 0 diagnóstico de morte encefálica e uma captação com mais segurança e qualidade (PESTANA AL, et al., 2013). É incontestável que a educação é um fator determinante para o sucesso ou insucesso do processo de doação e transplante, sendo os treinamentos, cursos e palestras estratégias fundamentais para instrumentalizar a assistência (MAGALHÃES ALP, et al., 2018; PESTANA AL, et al., 2013).

Nesta pesquisa verificou-se que $81,2 \%$ dos entrevistados consideram que faltam profissionais para 0 cuidado ao potencial doador, 64,9\% afirmam não estarem capacitados para a responsabilidade de cuidar do potencial doador e 65,9\% acreditam que faltam equipamentos para o cuidado do mesmo ou a falta de estrutura na instituição (61,2\%). Tais resultados corroboram com o estudo realizado em um hospital de grande porte na cidade de São Paulo, ao qual identificou-se que dentre as dificuldades vivenciadas por enfermeiros no processo de doação de órgãos estão o desconhecimento para a realização do protocolo de morte encefálica, a escassez de recursos humanos e dos materiais, bem como a falha na comunicação entre as equipes (ARAÚJO MN e MASSAROLLO MCKB, 2014). 
Para efetividade da doação de órgãos e tecidos é crucial a agilidade e precisão na condução do processo de doação, o que mais uma vez implica na importância de uma estrutura adequada, com recursos físicos e materiais apropriados e recursos humanos capacitados (FREIRE ILS, et al., 2014; PESTANA AL, et al., 2013).

Apesar de todos os desafios o COHREC concluiu $68,75 \%$ dos protocolos abertos no último biênio, e realizou 32 captações. Mais uma vez, esses dados evidenciam que a discrepância no que se refere ao processo de doação e transplante de órgãos. Visto que a demanda é desproporcional ao número de transplantes efetivados, o que tem resultado no crescimento das filas de espera (BASSO LD, et al., 2019).

No entanto, autores atribuem dois fatores para a redução da mortalidade na lista de espera dos transplantes: a organização e a efetiva participação das equipes multiprofissionais em todo o processo de doação (NOGUEIRA M, et al., 2017). Por fim, políticas de educação também se fazem necessárias, tanto para profissionais quanto para estudantes das áreas de saúde, além da população (PEGO-FERNANDES PM e GARCIA VD, 2010; SOUZA DRS, et al., 2019).

Freire SG et al. (2012), apontam ainda que, apesar da prevalência de internações de potenciais doadores ocorrer em salas de emergência, o cuidado prestado a esses pacientes deve dar-se, preferencialmente, em um ambiente de terapia intensiva. Portanto, considera-se o treinamento e motivação dos médicos de terapia intensiva como medidas para contribuir com o aumento no número de diagnósticos, uniformização nas condutas médicas e consequentemente a elevação nos índices de doações (PEGOFERNANDES PM e GARCIA VD, 2010; SOUZA DRS, et al., 2019).

\section{CONCLUSÃO}

Durante a pesquisa foi observado a dificuldade de encontrar estudos abordando essa temática na região, sendo relevante aprofundar estudos na área, a fim de contribuir para a formulação de políticas públicas voltadas a captação e ao transplante de órgãos, bem como, o fortalecimento da rede de atenção à saúde considerando estratégias que fomentem discussões entre profissional e sociedade. Outro ponto importante é a qualificação multiprofissional dentro dos setores de terapia intensiva e salas de emergência não só para diagnóstico e manutenção do potencial doador, mas para desenvolvimento de habilidades relacionadas a comunicação tanto de profissional para profissional, quanto de profissional para com os familiares. Além, de proporcionar ao profissional apoio psicológico e condições dignas de trabalho.

\section{REFERÊNCIAS}

1. ARAÚJO MN, MASSAROLLO MCKB. Conflitos éticos vivenciados por enfermeiros no processo de órgãos. Revista Acta Paulista de Enfermagem, 2014; 27(3):215-520.

2. ASSOCIAÇÃO BRASILEIRA DE TRANSPLANTE DE ÓRGÃOS - ABTO. Registro Brasileiro de Transplantes. Dimensionamento dos transplantes no Brasil e em cada estado (2010-2017). 2017; ano XXIII, no 4:104p.

3. ASSOCIAÇÃO BRASILEIRA DE TRANSPLANTE DE ÓRGÃOS - ABTO. Registro Brasileiro de Transplantes. Dimensionamento dos transplantes no Brasil e em cada estado (2011-2018). 2018; ano XXIV, no 4: 89p.

4. BASSO LD, et al. Dificuldades enfrentadas e condutas evidenciadas na atuação do enfermeiro frente à doação de órgãos: revisão integrativa. Revista Ciência, Cuidado e Saúde, 2019; 18(1):1-8.

5. BRASIL. Ministério da Saúde. Conselho Nacional de Saúde. Resolução n. 466, de 12 de dezembro de 2012. Diretrizes e normas regulamentadoras de pesquisa envolvendo seres humanos. Brasília, 2012.

6. CASTELLI I, JUNIOR ÁLC. Profissionais de saúde e o diagnóstico de morte encefálica: uma revisão. Revista Espacios, 2018; 39(7):6.

7. CIOATTO RM, PINHEIRO AAG. Transplante de órgãos humanos no Brasil: a temática não pode ser declarada morta. Revista de Direitos e Garantias Fundamentais, 2017; 18(3):177-214.

8. COELHO GHF, BONELLA AE. Doação de órgãos e tecidos humanos: a transplantação na Espanha e no Brasil. Revista Bioética, 2019; 27(3):419-429.

9. CONSELHO FEDERAL DE ENFERMAGEM - COFEN. Perfil da Enfermagem no Brasil. São Paulo, 2013; 32p.

10. CONSELHO FEDERAL DE MEDICINA - CFM. Resolução CFM № 2173, de 23 de Novembro de 2017. Define os critérios do diagnóstico de morte encefálica. Diário Oficial da União. Brasília, 2017. 
11. CUNHA DSP, et al. Morte encefálica e manutenção de órgãos: conhecimento dos profissionais intensivistas. Revista de Enfermagem UFPE online, 2018; 12(1):51-58.

12. DA EIRA CSL, TRINDADE MI. Doação de órgãos: a realidade de uma unidade de cuidados intensivos portuguesa. Revista Brasileira de Terapia Intensiva, 2018; 30(2):201-207.

13. FAGIOLI FGD, BOTONI FA. Tratamento do potencial doador de múltiplos órgãos. Revista Médica Minas Gerais, $2009 ; 19(3): 242-247$.

14. FREIRE SG, et al. Alterações fisiológicas da morte encefálica em potenciais doadores de órgãos e tecidos para transplantes. Escola Anna Nery Revista de Enfermagem, 2012; 16(4):761-766.

15. FREIRE ILS, et al. Facilitadores e barreiras na efetividade da doação de órgãos e tecidos. Revista Texto \& Contexto Enfermagem, 2014; 23(4):925-34.

16. HIRSCHHEIMER MR. Morte encefálica e doação de órgãos e tecidos. Revista Residência Pediátrica, 2016; 6(supl1):29-45.

17. JACAÚNA RO; DOS SANTOS TA, BRITO FPG. Conhecimento dos Acadêmicos de Enfermagem sobre o Processo de Doação de Órgãos e Tecidos para Transplante: uma Revisão de Literatura. In: Congresso Internacional de Enfermagem. 2017.

18. MAGALHÃES ALP, et al. Segurança do paciente no processo de doação e transplante de órgãos e tecidos. Cogitare Enfermagem, 2017; 22(2): e45621.

19. MAGALHÃES ALP, et al. Significados do cuidado de enfermagem ao paciente em morte encefálica potencial doador. Revista Gaúcha de Enfermagem, 2018; 39: e2017-0274.

20. NOGUEIRA M, et al. Doação de órgãos e tecidos para transplante: contribuições teóricas. Revista Recien - Revista Científica de Enfermagem, 2017; 7(20):58-69.

21. MORATO EG. Morte encefálica: conceitos essenciais, diagnóstico e atualização. Revista Médica Minas Gerais, $2009 ; 19(3): 227-236$.

22. PEGO-FERNANDES PM, GARCIA VD. Estado atual do transplante no Brasil. Revista Diagnóstico \& Tratamento, $2010 ; 15(2): 51-52$.

23. PESTANA AL, et al. Pensamento Lean e cuidado do paciente em morte encefálica no processo de doação de órgãos. Revista Escola de Enfermagem USP, 2013; 41(1):258-64.

24. REZENDE LBO, et al. Doação de órgãos no Brasil: uma análise das Campanhas Governamentais sob a perspectiva do marketing social. Revista Brasileira de Marketing, 2015; 14(3):362-376.

25. RIBEIRO SCP, et al. O conhecimento dos fisioterapeutas sobre morte encefálica e de sua atuação na manutenção de possíveis doadores. Revista Brasileira de Pesquisa em Saúde, 2017; 19(4):84-92.

26. RONDÔNIA. Secretária de Saúde do Estado de Rondônia - SESAU/RO. Complexo Hospitalar Regional de Cacoal - COHREC, Serviço de Arquivo Médico e Estatística - SAME, 2018.

27. RONDÔNIA. Secretária de Saúde do Estado de Rondônia - SESAU/RO. Complexo Hospitalar Regional de Cacoal - COHREC, Comissão Intra-Hospitalar de Doação de Órgãos e Tecidos para Transplante - CIHDOTT, 2018.

28. SOUZA DRS, TOSTES PP, SILVA AS. Morte encefálica: conhecimento e opinião dos médicos da Unidade de Terapia Intensiva. Revista Brasileira de Educação Médica, 2019; 43(3):115-22.

29. WESTPHAL GA, et al. Diretrizes para avaliação e validação do potencial doador de órgãos em morte encefálica. Revista Brasileira de Terapia Intensiva, 2016; 28(3):220-255. 